\title{
Dynamics and role of MicroRNAs during mammalian follicular development
}

\author{
S. Gebremedhn ${ }^{1}$, H.O. Pandey ${ }^{1}$, D. Salilew-Wondim ${ }^{1}$, M. Hoelker ${ }^{1,2,3}$, K. Schellander ${ }^{1,3}$, D. Tesfaye S $^{1,3,4}$ \\ ${ }^{1}$ Institute of Animal Science, Department of Animal Breeding and Husbandry, University of Bonn, Bonn, Germany. \\ ${ }^{2}$ Teaching and Research Station Frankenforst, Faculty of Agriculture, University of Bonn, Königswinter, Germany. \\ ${ }^{3}$ Center of Integrated Dairy Research, University of Bonn, Bonn, Germany.
}

\begin{abstract}
Ovarian functions, which involve dynamically regulated processes of selection, recruitment and dominance, are known to be regulated by an array of genes, which are expressed in spatiotemporal manner in follicular somatic cells and gametes. This differential expression of genes in mammalian follicular cells is partly regulated by posttranscriptional gene regulators named microRNAs (miRNAs). In addition to the cellular miRNAs, growing number of evidences are available on the potential role of extracellular vesicles mediated transfer of miRNAs in follicular fluid. These extracellular vesicles are shown to be involved in cellto-cell communication within the follicular environment. The molecular messages carried by the extracellular vesicles released into extracellular space are thought to be reflections of the physiological status of the cells from where they are released. Therefore due to their structural nature and potential to cargo several physiologically relevant molecules, exosomes have a great potential to be used as markers of oocyte developmental competence in follicular environment. Here, we review large sets of literatures to show the dynamic nature of miRNAs during various stages of mammalian follicular development, oocyte growth and the role of some of the miRNAs in ovarian cell functions. Moreover, the presence of microvesicle and exosome-coupled extracellular miRNAs in mammalian follicular fluid and their potential involvement in cellto-cell communication are briefly discussed.
\end{abstract}

Keywords: exosomes, extracellular vesicles, folliculogenesis, microRNAs, ovary.

\section{MicroRNAs in mammalian ovary}

The mammalian ovary is an organ where series of dynamically regulated processes of follicular recruitment, selection, dominance, ovulation and atresia undergo. These processes are under tight coordination of paracrine and endocrine factors which in turn regulate the expression and interaction of multitude of genes in the ovary (Hunter et al., 2004). In line with an attempt to unravel the genetic regulation of ovarian functions, the first functional importance of microRNAs (miRNAs) in female reproduction was evidenced by tissue specific knocking out of the Dicer 1; an important evolutionary conserved ribonuclease III enzyme involved in miRNAs biogenesis. The conditional inactivation of Dicerl in mouse ovarian granulosa, oviductal and uterine cells resulted in female infertility by decreasing the rate of ovulation, shortening the uterine horns and formation of oviductal cysts (Nagaraja et al., 2008). This has opened the door for further investigation of miRNAs and their role in mammalian ovaries. Subsequently, in the last decade intensive research on identification and functional analysis of miRNAs was performed in ovaries of various species. Several groups have reported that miRNAs are expressed in mammalian ovarian cells signifying their potential involvement in posttranscriptional regulation of important genes in the ovary. Construction of ovarian small RNAs complementary DNA (srcDNA) libraries revealed that miRNAs are the most abundantly expressed class of small RNAs in mouse ovary (Ro et al., 2007). A massive parallel sequencing of small RNA fraction extracted from newborn mouse ovary has identified 398 known and 4 novel microRNAs expressed in newborn mouse ovary (Ahn et al., 2010). Similarly, small RNA library constructed from cow ovary identified 50 known and 24 novel miRNAs, among which miRNAs; let-7a, let-7b, let-7c, miR-21, miR-23b, miR-24, miR-27a, miR-126 and miR-143 were found to be the most dominant ones (Hossain et al., 2009). The expression pattern of miRNAs in sheep ovaries during anestrus and the breeding season identified 97 known, 369 conserved and 17 predicted novel miRNAs (Di et al., 2014). Ovarian samples of uniparous and multiparous goats showed differential expression of miRNAs in which 8 miRNAs of the let-7 family; let-7b, let-7b-5p, let-7-5p, let-7c, let-7c-5p, let$7 \mathrm{f}-5 \mathrm{p}$, let-7f, let-7 and miR-140, miR-320a were the top 10 abundantly expressed miRNAs in both the uniparous and multiparous goat ovaries (Ling et al., 2014). Deep sequencing of miRNAs in porcine ovary also identified the abundance of around 732 mature miRNAs (Li et al., 2011). Following the identification of miRNAs in ovaries of various species, studies in the last years have focused on the expression characterization and functional analysis of these regulatory molecules in follicular growth and ovarian function.

\section{Expression dynamics of miRNAs during follicular development}

During the course of mammalian folliculogenesis, different miRNAs are expressed in theca cells, granulosa cells (Salilew-Wondim et al., 2014; Gebremedhn et al., 2015), cumulus-oocytecomplex (Abd El Naby et al., 2013), follicular-fluid (Sohel et al., 2013) and the corpus luteum (McBride et al., 2012; Maalouf et al., 2016b). We have previously shown significant differences in the expression of 
miRNAs in bovine granulosa cells derived from subordinate and dominant follicles of early luteal phase (day 3 and 7 of the estrous cycle; Salilew-Wondim et al., 2014) and late follicular phase (day 19 of the estrous cycle; Gebremedhn et al., 2015). Interestingly, the extent of differences in the relative abundance of miRNAs in granulosa cells of the two follicular categories was minimal at day 3 , whereas it was much pronounced at day 7 and 19. However, irrespective of the day of estrous cycle, miR-26a, miR-10b and the let7 family members were the most abundantly expressed miRNAs in granulosa cells of both dominant and subordinate follicles (Salilew-Wondim et al., 2014; Gebremedhn et al., 2015) signifying their cellular housekeeping role in bovine follicular development. The dynamic nature of miRNA expression has been also evidenced in ovine species during follicular-luteal transition in which miR-21, miR-125b, let-7a and let-7b were the most abundantly expressed miRNAs during comparative analysis of ovine growing follicles, preovulatory follicles, early and late corpus luteum (McBride et al., 2012). Similarly, microarray technology was used to investigate the association of miRNA expression with bovine follicular growth and atresia. For this, genome-wide miRNA expression analysis was performed on tissues samples derived from small growing $(4-8 \mathrm{~mm})$, large healthy $(12-17 \mathrm{~mm})$ and atretic follicles (Sontakke et al., 2014). Among the 17 differentially expressed miRNAs, miR-144, miR-202, miR-451, miR-652, and miR-873 were enriched in larger healthy follicles compared to the small growing follicles. Moreover, 57 miRNAs were differentially expressed between large healthy and large atretic follicles. To determine the involvement of selected miRNAs obtained from in vitro studies; miR-21, miR23a, miR-145, miR-503, miR-224, miR-383, miR-378, miR-132, and miR-212 in regulating equine follicular development, Donadeu and Schauer (2013) aspirated follicular fluid from dominant follicles during ovulatory and anovulatory seasons. It was shown that the relative levels of miR-21, miR-23b, miR-378 and miR-202 were higher in ovulatory follicles, whereas miR-145 tends to have higher level in anovulatory follicles. The level of miR-21, miR-132, miR-212, and miR-224 was increased in leading follicles, whereas the expression of their predicted target genes; PTEN, RASA1 and SMAD4 was reduced, indicating their potential involvement in regulating cell survival, differentiation and steroidogenesis during follicular selection in equine ovary (Schauer et al., 2013).

Depending on their genomic localization, miRNAs are grouped and termed as clusters (Mathelier and Carbone, 2013). MiRNA expression data analysis revealed an interesting pattern of co-enrichment or degradation of miRNA clusters in specific stages of bovine follicular development. Accordingly, the miR183-96-182 cluster, miR-132-212 cluster and miR-424450-542 cluster miRNAs were significantly enriched in granulosa cells of preovulatory dominant follicles while the miR-17-92 cluster miRNAs were enriched in granulosa cells of the subordinate follicles obtained at day 19 of the estrous cycle (Gebremedhn et al., 2015).

\section{Potential role of miRNAs in controlling ovarian functions}

During the course of follicular development, proliferation and differentiation of granulosa cells are essential for proper follicular recruitment, dominance, maturation, ovulation and atresia. Several studies have indicated the involvement of miRNAs in regulating the proliferation, differentiation, survival, cell cycle transition and/or apoptosis of granulosa cells (Carletti et al., 2010; Sirotkin et al., 2010; Yin et al., 2014; Andreas et al., 2016; Gebremedhn et al., 2016). Interestingly, in vitro granulosa cell culture models have become most popular and widely used to understand the potential role of miRNAs in ovarian functions. For this reason, majority of the functional studies of miRNAs in granulosa cells are performed through in vitro loss-andgain of function by modulating the levels of selected miRNAs (Maalouf et al., 2016a).

In an attempt to determine the role of human miR-15a in controlling basic granulosa cell functions, Sirotkin et al. (2014) transfected primary human granulosa cells with anti-miR-15a and miR-15a precursor followed by evaluation of the expression of marker genes for cell proliferation $(M A P K / E R K 1,2$ and $P C N A$ ), apoptosis (Caspase3 and Bax) and hormonal levels (Progesterone, Estradiol and Testosterone) in spent culture media. It was shown that inhibition of the miR-15a increased the protein accumulation of proliferation and apoptosis marker genes, reduction in the release of progesterone and testosterone and promotes the release of estradiol. Contrary to this, overexpression of miR-15a resulted in opposite effect. Evidences showed that multiple extra- and intraovarian factors such as the members of the TGF- $\beta$ superfamily members (van den Hurk and Zhao, 2005; Knight and Glister, 2006), activin receptor-like kinases (ALK) and the Smads (Miyazawa et al., 2002; Florio et al., 2010) are implicated in regulation of follicular development. Members of the TGF- $\beta$ superfamily, which are known to be involved in granulosa cell proliferation and differentiation (Hsueh et al., 1984; Hirshfield, 1991), were reported to be posttranscriptionally regulated by miRNAs in granulosa cells (Yao et al., 2014). In that study, over expression of miR-224 enhanced TGF- $\beta 1$ induced proliferation of granulosa cells by targeting Smad4 gene. Conversely, inhibition of miR-224 suppressed the proliferation of granulosa cells induced by TGF- $\beta 1$ treatment. Similarly, miR-181a expression decreased in mouse granulosa cells treated with activin $\mathrm{A}$ in dose dependent manner and overexpression of miR-181a inhibited cell proliferation by targeting acvr2a, which in turn resulted in suppression of CCND2 and PCNA (Zhang et al., 2013). Recently, we showed that the miR-183-96-182 cluster coordinately target the expression of $F O X O 1$ and promote proliferation and cell cycle transition of bovine granulosa cells. Overexpression of the miR-183-96-182 cluster miRNAs reduced the proportion of cells under $\mathrm{G} 0 / \mathrm{G} 1$ arrest and increased the proportion of cells entering the S-phase of the cell cycle. Interestingly, selective degradation of FOXO1 using siRNAs also promoted the cell 
proliferation and cell cycle transition. This evidence supports the idea of a coordinated role of this miRNA cluster in repressing anti-proliferation genes and their downstream transcripts (Gebremedhn et al., 2016). During various stages of ovarian follicular development, majority of the follicles undergo atresia being triggered by granulosa cells apoptosis (Portela et al., 2015). Accumulated evidences are available regarding the regulation of miRNAs in granulosa cells apoptosis and determine the follicular cells fate to either ovulation or atresia. In one of the early miRNA functional study, miR-21 as one of the three highly induced miRNAs in murine granulosa cells in response to ovulatory dose of LH administration, induces granulosa cells apoptosis upon its inhibition. In the same study, in vivo anti-miR21 treated ovary resulted in reduced rate of ovulation compared to the untreated contralateral ovary (Carletti et al., 2010). The let-7g induces apoptosis in pig granulosa cells by targeting the TGFBR1 gene (Zhou et al., 2015), while porcine atretic follicles are highly enriched with let- $7 \mathrm{~g}$ and its overexpression promoted apoptosis of granulosa cells (Cao et al., 2015). MiR-26b promotes porcine granulosa cells apoptosis and induces follicular atresia by targeting SMAD4 (Liu et al., 2014) and ATM (Lin et al., 2012). MiR-34a promotes granulosa cell apoptosis by targeting $I N H B B$ in porcine ovary (Tu et al., 2014). Similarly, functional analysis of the miR-17-92 cluster, which was found to be enriched in bovine granulosa cells derived from the subordinate follicles of day 19 of the estrous cycle, revealed the regulatory role of this cluster in granulosa cells proliferation, differentiation and steroidogenesis by targeting PTEN and BMPR2 genes. Overexpression of the miRNA cluster promoted cell proliferation, and decreased proportion of differentiating cells (Andreas et al., 2016).

\section{MiRNAs in steroidogenesis and their hormonal regulation}

According to the two-cell-two-gonadotropin theory, luteinizing hormone stimulates thecal cells to produce androgens, and follicle-stimulating hormone stimulates granulosa cells to produce estrogens from androgens (Barnes et al., 2000). Growing evidences indicate that miRNAs act as regulators of steroidogenesis (Schauer et al., 2013; Toms et al., 2015; Donadeu et al., 2016). The effect of miRNAs on the release of major ovarian steroid hormones was determined by transfecting human primary granulosa cells with 80 different human pre-miRNAs constructs, and the release of progesterone was inhibited by 36 miRNAs. Whereas, 10 miRNAs promoted progesterone release (Sirotkin et al., 2009). MiR-378 decreases ovarian estradiol production by targeting aromatase. However, overexpression of the aromatase 3'-UTR in granulosa cells enhanced aromatase expression at protein level, possibly mediated by the binding of miR378 within this region, thereby reducing the binding of this miRNA to the endogenous aromatase 3'-UTR (Xu et al., 2011). In a separate study, the progesterone receptor (PGR) was targeted by miR-378-3p which leads to decrease in the protein and mRNA levels and its downstream target genes; ADAMTS1, CTSL1, and $P P A R G$, which are involved in follicular maturation and remodeling (Toms et al., 2015). A recent study showed that level of miR-378 in the follicular wall was correlated with estradiol, the estradiol: progesterone ratio and CYP19A1 (Donadeu et al., 2016). MiR-34a and miR-320 inhibit estradiol release from human granulosa cells and murine ovaries, respectively (Sirotkin et al., 2009; Yao et al., 2014). However, miR320 could stimulate testosterone and progesterone in murine ovaries. Interestingly, miR-383 has been shown to act as positive regulator of estradiol production in mouse granulosa cells by inhibiting RBMS1, which leads to c-Myc inactivation and steroidogenesis (Yin et al., 2014). Moreover, miR-132 is involved in the cAMP signaling pathway and promotes estradiol synthesis via the translational repression of Nurrl in ovarian granulosa cells and significantly induce expression of Cyp19al through Nurr1; a direct target that suppresses Cyp19a1 expression (Wu et al., 2015). Similarly, miR$133 \mathrm{~b}$ is involved in follicle-stimulating hormone (FSH)induced estrogen production by down-regulating Foxl2 and inhibiting the Foxl2-mediated transcriptional repression of StAR and CYP19A1 to promote estradiol production (Dai et al., 2013). The overexpression of miR-20a, which is validated to target PTEN and BMPR2 genes, induced the release of progesterone in in vitro bovine granulosa cell culture (Andreas et al., 2016)

Recently, we investigated the effect of supraphysiological level of gonadotropins introduction during the process of controlled ovarian hyperstimulation on the level of extracellular miRNAs in bovine follicular fluid and blood plasma (Noferesti et al., 2015). It was shown that, a total of 57 and 21 miRNAs were differentially expressed in follicular fluid and blood plasma, respectively, derived from hyper-stimulated heifers compared to the unstimulated control counterpart. Bioinformatics analysis revealed that pathways including the TGF- $\beta$ signaling, MAPK signaling and oocyte meiosis are significantly enriched by target genes of the miRNAs selectively upregulated in follicular fluid and blood plasma of hyper-stimulated heifers (Noferesti et al., 2015). Despite the fact that the choice of the mechanism of release of miRNAs into extracellular space is not understood well, we have detected circulatory miRNAs in exosomes and Ago2 fraction of follicular fluid and blood plasma of hyperstimulated and unstimulated control heifers. This may evidence the releasing mechanism of miRNAs into extracellular space in both follicular fluid and blood plasma was not affected by super-stimulation treatment.

\section{Extracellular miRNAs in follicular fluid}

While the majority of miRNAs are found intracellularly, tremendous numbers of miRNAs have been found to circulate in the extracellular space and biological fluids, including follicular fluid (Donadeu and Schauer, 2013; Sohel et al., 2013; Santonocito et al., 2014). Mammalian follicular fluid consists of a 
complex mixture of nucleic acids, proteins, metabolites and ions, which are known to be secreted by the oocyte, granulosa cells, theca cells and blood plasma components that come to the follicular fluid via theca capillaries (Revelli et al., 2009; Rodgers and IrvingRodgers, 2010). The follicular fluid creates very suitable microenvironment for the growth and development of oocytes and its biochemical composition varies depending on the physiological status of the follicle. As any alteration in the follicular fluid composition can be associated with the oocyte quality, the follicular fluid components may provide useful diagnostic information on oocyte developmental competence and ovarian functions (Revelli et al., 2009). Several biochemical components including hormones, growth factors, cytokines and chemokines, which are secreted by follicular cells into follicular fluid, are known to promote oocyte maturation. Evaluation of any of specific components within the follicular fluid at any stage of follicular development revealed the physiological status of the animal in general and the oocyte health or growth status in particular (Sohel et al., 2013). In addition to autocrine and paracrine mode of communication inside the ovarian follicle, recently alternative mechanisms come into play. This mechanism in which the oocytes and follicular somatic cells exchange signals is mediated by the so called microvesicles and exosomes carrying RNAs (mRNA, miRNA, IncRNA and other types of RNAs), proteins and DNA fragments.

Extracellular vesicles can be broadly classified into 3 main classes: Microvesicles/ microparticles/ ectosomes, which are produced by outward budding and fusion of the plasma membrane, whereas exosomes are formed within the endosomal network and released upon fusion of multivesicular bodies with the plasma membrane. The release of extracellular vesicles (EVs), including exosomes and microvesicles, is a phenomenon shared by many cell types as a means of communicating with other cells. EVs released into the extracellular space can potentially reach distant tissues via circulation and once up taken by neighboring and/or distant cells, EVs can transfer functional molecules that may alter the status of recipient cells, thereby contributing to both physiological and pathological processes.

Extracellular microvesicles and exosomes in follicular fluid carrying miRNAs as cargo molecules have been detected in bovine (Sohel et al., 2013; Noferesti et al., 2015; Navakanitworakul et al., 2016), equine (da Silveira et al., 2012, 2014, 2015) and human (Xiao et al., 2016). The presence of EVs (microvesicles or exosomes) in follicular fluid may support the notion that they play a significant role in ovarian functions. The size and concentration of EVs in the follicular fluid between different sized follicles has been investigated (Navakanitworakul et al., 2016). In the same study while no significant changes were observed in the size of the extracellular vesicles between small, medium and large follicles, the concentration of extracellular vesicles decreased progressively as the follicle size increases. A microarray or sequencing based expression profiling of exosomes from bovine follicular fluid identified miRNAs enriched in follicular size dependent manner (Navakanitworakul et al., 2016) and oocyte developmental competence (Sohel et al., 2013). Interestingly, in the former study those miRNAs enriched in exosomes isolated from small follicle were found to be associated with cell proliferation, while miRNAs abundant in large follicle were found to be related to inflammatory response pathways. Similarly, significant number of miRNAs have been identified to be differentially carried by exosomes or Ago 2 protein complexes isolated from follicular fluid harboring oocytes with different growth status (Sohel et al., 2013). Those differentially expressed miRNAs from both exosomal and non-exosomal fraction of the follicular fluid were found to be involved in molecular pathways (MAPK signaling pathway, focal adhesion and regulation of actin cytoskeleton), which are known to be main regulators of follicular development and oocyte growth.

During the course of bovine follicular development, expansion of cumulus cells is essential for oocyte maturation and release through ovulation for a successful fertilization and early embryo development. LH induced increase in the expression of genes such as prostaglandin-endoperoxide synthase 2 (Ptgs2), pentraxin-related protein 3 (Ptx3), and tumor necrosis factor alpha-induced protein 6 (Tnfaip6) are essential for the expansion of cumulus cells. Recently, coincubation of bovine COC with extracellular vesicles isolated from follicular fluid aspirated from small and large follicles under in vitro condition resulted in cumulus expansion and increased expression of the aforementioned genes (Hung et al., 2015). Moreover, age related differences in exosome mediated miRNA abundance in follicular fluid of mare has been evidenced (da Silveira et al., 2015). This differential expression of exosomal miRNAs has been associated with the expression of granulosa cells genes associated with TGF- $\beta$ signaling pathway, which is the most dominant pathway modulating follicular development and oocyte growth.

\section{Potential role of extracellular miRNAs in cell-to-cell communication}

Ovarian follicular development and oocyte maturation are well coordinated processes and mediated by a constant exchange of signals between ovarian somatic cells and the oocytes (Zuccotti et al., 2011). These exchanges of signals are made possible through the establishment of gap junctions between the surrounding somatic cells and the oocytes (Cecconi et al., 2004; Gilchrist et al., 2008). In the follicular environment the physical contact between the surrounding cells especially granulosa and oocytes is mediated by the expression of key molecules mainly connexines ( $C X 37$ and $C X 43)$ which are also expressed in early stages of mammalian embryo development (Gittens and Kidder 2005; Gittens et al., 2005). Mutual communication between the oocyte and cumulus cells is achieved through secretion of both oocyte or cumulus 
cells secreted factors and are shown to affect each other in relative abundance of transcripts at mRNA (Regassa et al., 2011) and miRNA level (Abd El Naby et al., 2013). The oocyte secreted factors which pass through the gap junctions include ions, metabolites and amino acids that are necessary for oocyte growth, as well as small regulatory molecules that control oocyte development (Gilchrist et al., 2008). In addition to the direct communication, the transfer of signals between the different follicular cell types could be facilitated by extracellular vesicles circulating in the follicular fluid.

The release of EVs, including exosomes and microvesicles, is a cellular characteristics shared by many cell types as a means of communication with other cells and also potentially removing cell contents. This phenomenon is more evident in follicular environment where cell-to-cell communication is vital for the growth of the oocytes. MiRNAs carried by microvesicles and exosomes in follicular fluid are also found to be present in the surrounding follicular cells including theca cells, granulosa cells and cumulusoocyte-complex (Sohel et al., 2013). In addition to the identification of miRNAs carried by exosomes in mammalian follicular fluid, several in vitro experiments have been conducted to simulate the cell-to-cell communication mediated by exosomes (da Silveira et al., 2012, 2014; Sohel et al., 2013; Di Pietro, 2016). For this, we have previously shown that membrane labeled exosomes isolated from bovine follicular fluid harboring either growing or fully grown oocytes, were co-cultured with granulosa cells in vitro (Sohel et al., 2013). The uptake of exosomes by cultured granulosa cells and resulted in subsequent increase in endogenous cellular miRNAs and altered gene expression. Similar experiment in equine has also evidenced the uptake of microvesicles by cultured granulosa cells in vitro and in vivo experiments (da Silveira et al., 2012). Exosomes isolated from mid-estrous and preovulatory follicles and known to cargo $A C V R 1$ and miR-27b, miR-372, and miR-382 were found to alter the ID2 (an ACVR1 target gene) in preovulatory mare follicle (da Silveira et al., 2014).

All in all, the follicular fluid will provide a potential environment for identification of marker molecules associated with follicular development and oocyte competence. Moreover, with the identification of extracellular vesicles as cargo for molecular signals, tremendous opportunities will be opened for therapeutics in the field of fertility treatments.

\section{Conclusion}

Mammalian folliculogenesis or ovarian functionality is well coordinated process involving a molecular cross-talk between the various somatic cell types and the developing gamete in the follicular microenvironment to attain successful ovulation, fertilization and development of embryo and to give birth to healthy offspring. Understanding the genetic regulation of these processes paves the way to identify molecular profiles and their regulatory mechanisms associated with ovarian function and facilitates the development of diagnostic markers for oocyte developmental competence. Especially, exploring follicular fluid for the presence of extracellular miRNAs, which could be indicators of ovarian functions and subsequently competence of oocytes, facilitates the development of non-invasive diagnostic tools to address female infertility. Moreover, future researches need to focus in deciphering the exact sources and role extracellular miRNAs in follicular fluid towards the development of therapeutic tools for fertility treatments associated with ovarian disorders.

\section{References}

Abd El Naby WS, Hagos TH, Hossain MM, SalilewWondim D, Gad AY, Rings F, Cinar MU, Tholen E, Looft C, Schellander K, Hoelker M, Tesfaye D. 2013. Expression analysis of regulatory microRNAs in bovine cumulus oocyte complex and preimplantation embryos. Zygote, 21:31-51.

Ahn HW, Morin RD, Zhao H, Harris RA, Coarfa C, Chen ZJ, Milosavljevic A, Marra MA, Rajkovic A. 2010. MicroRNA transcriptome in the newborn mouse ovaries determined by massive parallel sequencing. $\mathrm{Mol}$ Hum Reprod, 16:463-471.

Andreas E, Hoelker M, Neuhoff C, Tholen E, Schellander K, Tesfaye D, Salilew-Wondim D. 2016. MicroRNA 17-92 cluster regulates proliferation and differentiation of bovine granulosa cells by targeting PTEN and BMPR2 genes. Cell Tissue Res. doi:10.1007/s00441-016-2425-7.

Barnes RB, Rosenfield RL, Namnoum A, Layman LC. 2000. Effect of follicle-stimulating hormone on ovarian androgen production in a woman with isolated follicle-stimulating hormone deficiency. $N$ Engl J Med, 343:1197-1198.

Cao R, Wu WJ, Zhou XL, Xiao P, Wang Y, Liu HL. 2015. Expression and preliminary functional profiling of the let-7 family during porcine ovary follicle atresia. Mol Cells, 38:304-311.

Carletti MZ, Fiedler SD, Christenson L,K. 2010. MicroRNA 21 blocks apoptosis in mouse periovulatory granulosa cells. Biol Reprod, 83:286-295.

Cecconi S, Ciccarelli C, Barberi M, Macchiarelli G, Canipari R. 2004. Granulosa cell-oocyte interactions. Eur J Obstet Gynecol Reprod Biol, 1:S19-22.

da Silveira JC, Veeramachaneni DN, Winger QA, Carnevale EM, Bouma GJ. 2012. Cell-secreted vesicles in equine ovarian follicular fluid contain miRNAs and proteins: a possible new form of cell communication within the ovarian follicle. Biol Reprod, $86: 71$.

da Silveira JC, Carnevale EM, Winger QA, Bouma GJ. 2014. Regulation of ACVR1 and ID2 by cellsecreted exosomes during follicle maturation in the mare. Reprod Biol Endocrinol, 12:44.

da Silveira JC, Winger QA, Bouma GJ, Carnevale EM. 2015. Effects of age on follicular fluid exosomal microRNAs and granulosa cell transforming growth factor-? signalling during follicle development in the mare. Reprod Fertil Dev, 27:897-905.

Dai A, Sun H, Fang T, Zhang Q, Wu S, Jiang Y, 
Ding L, Yan G, Hu Y. 2013. MicroRNA-133b stimulates ovarian estradiol synthesis by targeting Fox12. FEBS Lett, 587:2474-2482.

Di Pietro C. 2016. Exosome-mediated communication in the ovarian follicle. J Assist Reprod Genet, 33:303311.

Di R, He J, Song S, Tian D, Liu Q, Liang X, Ma Q, Sun M, Wang J, Zhao W, Cao G, Yang Z, Ge Y, Chu M. 2014. Characterization and comparative profiling of ovarian microRNAs during ovine anestrus and the breeding season. BMC Genomics, 15:1471-2164.

Donadeu FX, Schauer SN. 2013. Differential miRNA expression between equine ovulatory and anovulatory follicles. Domest Anim Endocrinol, 45:122-125.

Donadeu FX, Sontakke SD, Ioannidis J. 2016. MicroRNA indicators of follicular steroidogenesis. Reprod Fertil Dev. doi: 10.1071/RD15282.

Florio P, Gabbanini M, Borges LE, Bonaccorsi L, Pinzauti S, Reis FM, Boy Torres P, Rago G, Litta P, Petraglia F. 2010. Activins and related proteins in the establishment of pregnancy. Reprod Sci, 17:320-330.

Gebremedhn S, Salilew-Wondim D, Ahmad I, Sahadevan S, Hossain MM, Hoelker M, Rings F, Neuhoff C, Tholen E, Looft C, Schellander K, Tesfaye D. 2015. MicroRNA expression profile in bovine granulosa cells of preovulatory dominant and subordinate follicles during the late follicular phase of the estrous cycle. PLoS One, 10:e0125912.

Gebremedhn S, Salilew-Wondim D, Hoelker M, Rings F, Neuhoff C, Tholen E, Schellander K, Tesfaye D. 2016. MicroRNA-183 96 182 cluster regulate bovine granulosa cell proliferation and cell cycle transition by coordinately targeting FOXO1. Biol Reprod, 94:127.

Gilchrist RB, Lane M, Thompson JG. 2008. Oocytesecreted factors: regulators of cumulus cell function and oocyte quality. Hum Reprod Update, 14:159-177.

Gittens JE, Barr KJ, Vanderhyden BC, Kidder GM. 2005. Interplay between paracrine signaling and gap junctional communication in ovarian follicles. $J$ Cell Sci, 118:113-122.

Gittens JE, Kidder GM. 2005. Differential contributions of connexin37 and connexin43 to oogenesis revealed in chimeric reaggregated mouse ovaries. J Cell Sci, 118:5071-5078.

Hirshfield AN. 1991. Development of follicles in the mammalian ovary. Int Rev Cytol, 124:43-101.

Hossain MM, Ghanem N, Hoelker M, Rings F, Phatsara C, Tholen E, Schellander K, Tesfaye D. 2009. Identification and characterization of miRNAs expressed in the bovine ovary. BMC Genomics, 10:1471-2164.

Hsueh AJ, Adashi EY, Jones PB, Welsh TH Jr. 1984. Hormonal regulation of the differentiation of cultured ovarian granulosa cells. Endocr Rev, 5:76-127.

Hung WT, Hong X, Christenson LK, McGinnis LK. 2015. Extracellular vesicles from bovine follicular fluid support cumulus expansion. Biol Reprod, 93:1-9.

Hunter MG, Robinson RS, Mann GE, Webb R. 2004. Endocrine and paracrine control of follicular development and ovulation rate in farm species. Anim Reprod Sci, 83:461-477.
Knight PG, Glister C. 2006. TGF-beta superfamily members and ovarian follicle development. Reproduction, 132:191-206.

Li M, Liu Y, Wang T, Guan J, Luo Z, Chen H, Wang X, Chen L, Ma J, Mu Z, Jiang AA, Zhu L, Lang Q, Zhou X, Wang J, Zeng W, Li N, Li K, Gao X, Li X. 2011. Repertoire of porcine microRNAs in adult ovary and testis by deep sequencing. Int J Biol Sci, 7:1045-1055.

Lin F, Li R, Pan ZX, Zhou B, Yu de B, Wang XG, Ma XS, Han J, Shen M, Liu HL. 2012. miR-26b promotes granulosa cell apoptosis by targeting ATM during follicular atresia in porcine ovary. PLoS One, 7:e38640.

Ling YH, Ren CH, Guo XF, Xu LN, Huang YF, Luo JC, Zhang YH, Zhang XR, Zhang ZJ. 2014. Identification and characterization of microRNAs in the ovaries of multiple and uniparous goats (Capra hircus) during follicular phase. BMC Genomics, 15:1471-2164.

Liu J, Du X, Zhou J, Pan Z, Liu H, Li Q. 2014. MicroRNA-26b functions as a proapoptotic factor in porcine follicular granulosa cells by targeting Sma-and Mad-related protein 4. Biol Reprod, 91:146.

Maalouf SW, Liu WS, Pate JL. 2016a. MicroRNA in ovarian function. Cell Tissue Res, 363:7-18.

Maalouf SW, Smith CL, Pate JL. 2016b. Changes in MicroRNA Expression during maturation of the bovine corpus luteum: regulation of luteal cell proliferation and function by microRNA-34a. Biol Reprod, 94:10.

Mathelier A, Carbone A. 2013. Large scale chromosomal mapping of human microRNA structural clusters. Nucleic Acids Res, 41:4392-4408.

McBride D, Carre W, Sontakke SD, Hogg CO, Law A, Donadeu FX, Clinton M. 2012. Identification of miRNAs associated with the follicular-luteal transition in the ruminant ovary. Reproduction, 144:221-233.

Miyazawa K, Shinozaki M, Hara T, Furuya T, Miyazono K. 2002. Two major Smad pathways in TGF-beta superfamily signalling. Genes Cells, 7:11911204.

Nagaraja AK, Andreu-Vieyra C, Franco HL, Ma L, Chen R, Han DY, Zhu H, Agno JE, Gunaratne PH, DeMayo FJ, Matzuk MM. 2008. Deletion of Dicer in somatic cells of the female reproductive tract causes sterility. Mol Endocrinol, 22:2336-2352.

Navakanitworakul R, Hung WT, Gunewardena S, Davis JS, Chotigeat W, Christenson LK. 2016. Characterization and small RNA content of extracellular vesicles in follicular fluid of developing bovine antral follicles. Sci Rep 6:25486.

Noferesti SS, Sohel MMH, Hoelker M, SalilewWondim D, Tholen E, Looft C, Rings F, Neuhoff C, Schellander K, Tesfaye D. 2015. Controlled ovarian hyperstimulation induced changes in the expression of circulatory miRNA in bovine follicular fluid and blood plasma. J Ovarian Res, 8:1-16.

Portela VM, Dirandeh E, Guerrero-Netro HM, Zamberlam G, Barreta MH, Goetten AF, Price CA. 2015. The role of fibroblast growth factor-18 in follicular atresia in cattle. Biol Reprod, 92:14.

Regassa A, Rings F, Hoelker M, Cinar U, Tholen E, Looft C, Schellander K, Tesfaye D. 2011. 
Transcriptome dynamics and molecular cross-talk between bovine oocyte and its companion cumulus cells. BMC Genomics, 12:57.

Revelli A, Delle Piane L, Casano S, Molinari E, Massobrio M, Rinaudo P. 2009. Follicular fluid content and oocyte quality: from single biochemical markers to metabolomics. Reprod Biol Endocrinol, 7:1477-7827.

Ro S, Song R, Park C, Zheng H, Sanders KM, Yan W. 2007. Cloning and expression profiling of small RNAs expressed in the mouse ovary. $R N A, 13: 2366-2380$.

Rodgers RJ, Irving-Rodgers HF. 2010. Formation of the ovarian follicular antrum and follicular fluid. Biol Reprod, 82:1021-1029.

Salilew-Wondim D, Ahmad I, Gebremedhn S, Sahadevan S, Hossain MD, Rings F, Hoelker M, Tholen E, Neuhoff C, Looft C, Schellander K, Tesfaye D. 2014. The expression pattern of microRNAs in granulosa cells of subordinate and dominant follicles during the early luteal phase of the bovine estrous cycle. PLoS One, 9:e106795.

Santonocito M, Vento M, Guglielmino MR, Battaglia R, Wahlgren J, Ragusa M, Barbagallo $D$, Borzi $P$, Rizzari S, Maugeri M, Scollo P, Tatone C, Valadi H, Purrello M, Di Pietro C. 2014. Molecular characterization of exosomes and their microRNA cargo in human follicular fluid: bioinformatic analysis reveals that exosomal microRNAs control pathways involved in follicular maturation. Fertil Steril, 102:1751-1761.e1.

Schauer SN, Sontakke SD, Watson ED, Esteves CL, Donadeu FX. 2013. Involvement of miRNAs in equine follicle development. Reproduction, 146:273-282.

Sirotkin AV, Ovcharenko D, Grossmann R, Laukova M, Mlyncek M. 2009. Identification of microRNAs controlling human ovarian cell steroidogenesis via a genome-scale screen. J Cell Physiol, 219:415-420.

Sirotkin AV, Laukova M, Ovcharenko D, Brenaut P, Mlyncek M. 2010. Identification of microRNAs controlling human ovarian cell proliferation and apoptosis. J Cell Physiol, 223:49-56.

Sirotkin AV, Kisova G, Brenaut P, Ovcharenko D, Grossmann R, Mlyncek M. 2014. Involvement of microRNA Mir15a in control of human ovarian granulosa cell proliferation, apoptosis, steroidogenesis, and response to FSH. Microrna, 3:29-36.

Sohel MM, Hoelker M, Noferesti SS, SalilewWondim D, Tholen E, Looft C, Rings F, Uddin MJ, Spencer TE, Schellander K, Tesfaye D. 2013. Exosomal and non-exosomal transport of extra-cellular microRNAs in follicular fluid: implications for bovine oocyte developmental competence. PLoS One 8:e78505. Sontakke SD, Mohammed BT, McNeilly AS, Donadeu FX. 2014. Characterization of microRNAs differentially expressed during bovine follicle development. Reproduction 148:271-283.

Toms D, Xu S, Pan B, Wu D, Li J. 2015. Progesterone receptor expression in granulosa cells is suppressed by microRNA-378-3p. Mol Cell Endocrinol, 399:95-102.

Tu F, Pan ZX, Yao Y, Liu HL, Liu SR, Xie Z, Li QF. 2014. miR-34a targets the inhibin beta $B$ gene, promoting granulosa cell apoptosis in the porcine ovary. Genet Mol Res, 13:2504-2512.

van den Hurk R, Zhao J. 2005. Formation of mammalian oocytes and their growth, differentiation and maturation within ovarian follicles. Theriogenology, 63:1717-1751.

Wu S, Sun H, Zhang Q, Jiang Y, Fang T, Cui I, Yan G, Hu Y. 2015. MicroRNA-132 promotes estradiol synthesis in ovarian granulosa cells via translational repression of Nurr1. Reprod Biol Endocrinol, 13:94.

Xiao GY, Cheng CC, Chiang YS, Cheng WT, Liu IH, Wu SC. 2016. Exosomal miR-10a derived from amniotic fluid stem cells preserves ovarian follicles after chemotherapy. Sci Rep, 6:23120.

Xu S, Linher-Melville K, Yang BB, Wu D, Li J. 2011. Micro-RNA378 (miR-378) regulates ovarian estradiol production by targeting aromatase. Endocrinology, 152:3941-3951.

Yao G, Liang M, Liang N, Yin M, Lu M, Lian J, Wang Y, Sun F. 2014. MicroRNA-224 is involved in the regulation of mouse cumulus expansion by targeting Ptx3. Mol Cell Endocrinol, 382:244-253.

Yin M, Wang X, Yao G, Lu M, Liang M, Sun Y, Sun F. 2014. Transactivation of micrornA-320 by microRNA-383 regulates granulosa cell functions by targeting E2F1 and SF-1 proteins. J Biol Chem, 289:18239-18257.

Zhang Q, Sun H, Jiang Y, Ding L, Wu S, Fang T, Yan G, Hu Y. 2013. MicroRNA-181a suppresses mouse granulosa cell proliferation by targeting activin receptor IIA. PLoS One, 8:e59667.

Zhou J, Liu J, Pan Z, Du X, Li X, Ma B, Yao W, Li Q, Liu H. 2015. The let-7g microRNA promotes follicular granulosa cell apoptosis by targeting transforming growth factor-beta type 1 receptor. Mol Cell Endocrinol, 409:103-112.

Zuccotti M, Merico V, Cecconi S, Redi CA, Garagna S. 2011. What does it take to make a developmentally competent mammalian egg? Hum Reprod Update, 17:525-540. 